\title{
Safety and efficacy of alendronate to treat osteopenia in children during therapy for acute lymphoblastic leukemia. A retrospective cohort study of sequential outcomes.
}

\author{
Ronald Barr ${ }^{1}$, Paula MacDonald ${ }^{2}$, Amy Cranston ${ }^{2}$, Misha Virdee ${ }^{3}$, Troy Farncombe ${ }^{4}$, and \\ Uma Athale ${ }^{3}$ \\ ${ }^{1}$ McMaster Children's Hospital \\ ${ }^{2}$ Hamilton Health Sciences \\ ${ }^{3}$ McMaster University \\ ${ }^{4}$ hamilton Health Sciences
}

February 7, 2022

\begin{abstract}
Background. Low bone mineral density (osteopenia) is encountered in children with acute lymphoblastic leukemia (ALL) before, during and after treatment. Prior experience with alendronate, an oral bisphosphonate, demonstrated high tolerability and evident clinical efficacy. However, concerns have been expressed about the long-term safety and utility of such agents in children. Procedure. Of 217 children with ALL treated on Dana Farber Cancer Institute protocols 69 received alendronate for a mean of 87 weeks after dual energy X ray absorptiometry (DXA). DXA was repeated following completion of alendronate, and again 5-9 years later in a subgroup of 32 children. Lumbar spine areal bone mineral (LS aBMD) Z scores were obtained and values corrected for height, age and weight (HAW) were calculated for subjects 3-18 years of age. Results. Almost 80\% $(\mathrm{N}=172)$ of the children remain in continuous complete remission at a mean of 14.5 years from diagnosis. Of those who receive alendronate, which was almost uniformly well tolerated, 7/69 (10.3\%) relapsed compared to 19/89 (21.3\%) who did not receive the drug. The mean unmodified LS aBMD Z score rose from -1.78 to -0.47 . This gain was statistically significant for both unmodified ( $\mathrm{p}<0.0001$ ) and HAW corrected $\mathrm{Z}$ scores -1.32 to $-0.42 ; \mathrm{p}<0.0001$ ). There was a modest median loss of LS aBMD ( $\mathrm{Z}$ score 0.045$)$ subsequently in the subgroup $(\mathrm{N}=32)$ of subjects on long-term follow up. Discussion. Alendronate appears to be well tolerated and moderately effective in osteopenic children with ALL. Whether it offers protection against relapse of leukemia needs further study.
\end{abstract}

\section{Introduction}

Bony morbidity is common in children with acute lymphoblastic leukemia (ALL). Pain is a frequent symptom at diagnosis, ${ }^{1}$ associated with an expanding cell mass in the medullary cavity, and vertebral fractures at this time are prominent and often unrecognized clinically. ${ }^{2}$ Osteopenia, reflecting loss of bone mineral, is demonstrable before the initiation of therapy ${ }^{3}$ and becomes more evident with the onset of treatment. ${ }^{4}$ The contributing factors include glucocorticoid and methotrexate medication, cranial irradiation (now used much less frequently) and reduced physical activity. ${ }^{5}$

Additional bony morbidity is seen in the form of osteonecrosis that shares an etiology with osteopenia, from exposure to high cumulative doses of corticosteroids, although the pathogenesis is different. ${ }^{6}$ Both osteopenia ${ }^{7}$ and the symptoms of osteonecrosis ${ }^{8}$ may be ameliorated by the administration of bisphosphonates.

These compounds are structural analogues of natural inorganic pyrophosphate which explains their very high affinity for bone because they bind to crystals of hydroxyapatite and impede their breakdown, so suppressing 
bone resorption. ${ }^{9}$ The third generation bisphosphonates, including alendronate and pamidronate, have side chains containing nitrogen. These agents bind to and inhibit farnesyl pyrophosphate synthase, an important enzyme in the mevalonic acid pathway. In turn this results in dysregulation of osteoclast metabolism with eventual apoptosis. ${ }^{9}$ Bone resorption remains suppressed for the duration of treatment. With oral agents only about $50 \%$ of the absorbed dose is retained in the skeleton, the remainder being excreted in the urine. The amount of bisphosphonate retained varies considerably among patients and between clinical disorders, probably reflecting variations in bone turnover. ${ }^{8}$

In our preliminary experience from 2000, intravenous pamidronate was effective in redressing the depletion of bone mineral but poorly tolerated; ${ }^{10}$ however, oral alendronate, with 5 fold greater potency, ${ }^{9}$ was well tolerated and retained clinical efficacy, ${ }^{11}$ despite low bioavailability. ${ }^{12}$

Nonetheless, concerns have been expressed about the safety and efficacy of bisphosphonates in children. ${ }^{13}$ While there have been no reports in childhood of the uncommon but dramatic problem of osteonecrosis of the jaw encountered in adults, ${ }^{14}$ even in those children undergoing invasive dental procedures while receiving a bisphosphonate ${ }^{15}$ the long-term impact of these drugs on growing bones is uncertain. ${ }^{16}$ Likewise, although bisphosphonates may exert anti-tumor effects, ${ }^{17,18}$ minor hematological abnormalities have been described with the administration of these agents ${ }^{19}$ and longer term adverse sequelae on hematopoiesis should be considered, including relapsed disease. Consequently, we have undertaken studies of the short and long-term efficacy and safety of bisphosphonates in children with ALL.

\section{Patients and Methods}

Between January, 2000 and April, 2015 a total of 223 children and adolescents were diagnosed with ALL at McMaster Children's Hospital (MCH) in Hamilton, Canada. Six of them were infants, less than 12 months of age, who received very intensive chemotherapy according to Interfant protocols ${ }^{20}$ and were excluded from this study. Among the 9 early deaths, 5 occurred during remission induction (2.3\%) and one child was withdrawn from treatment by her family at the end of induction while another returned to his native country at the same juncture. The study sample of 217 were treated on regimens $00-001^{, 21} 05-001^{22}$ and $11-001^{23}$ of the Dana Farber Cancer Institute Childhood ALL Consortium in which MCH had been a member since 1985. These regimens were characterized by high cumulative doses of glucocorticosteroids and asparaginase. Details of their disease and clinical outcomes are given in Table 1. For the purposes of this report the study sample was followed until December 31, 2020.

It was our standard practice, in the time period 2000-2015, to undertake dual energy x-ray absorptiometry (DXA) on all children $>3$ years of age at diagnosis and at intervals of 6 months thereafter for the duration of treatment; two years after remission induction ${ }^{24}$. These examinations were performed with Hologic densitometers QDR 4500A and Discovery A (Hologic Inc., Waltham, MA). Each whole body scan results in radiation exposure of 20 microSv, less than $1 / 10^{\text {th }}$ of a chest x-ray. ${ }^{25}$ Local normative data on body composition, ${ }^{26}$ including bone mineral density (BMD), ${ }^{27}$ were generated for $3-18$ year olds and corrections for height, age and weight (HAW) were developed for BMD of the lumbar spine. ${ }^{28}$

Children and adolescents with lumbar spine areal BMD (LS aBMD) Z scores of $<-2.0$ and those who were less osteopenic but had related bony morbidity, e.g. fractures, were candidates for treatment with a bisphosphonate, oral alendronate in all of them. Alendronate was administered weekly, in doses according to body weight (Table 2), to coincide with scheduled clinic attendance for chemotherapy, and all patients received daily supplemental calcium in the form of TUMS to provide 30 to $40 \mathrm{mg} / \mathrm{kg} / \mathrm{d}$. Alendronate was administered while the children were fasting, as recommended. ${ }^{12}$

The study was approved by the Hamilton Integrated Research Ethics Board, project 3479 C.

\section{Statistical analyses}

Patient demographics including BMD were summarized using descriptive measures expressed as mean (standard deviation) or median (minimum, maximum) for continuous variables and number (percent) for categorical variables. Associations between categorical outcomes and clinical groups were assessed using Fisher's 
Exact test or chi-squared test. Impact of bisphosphonate therapy on BMD was tested using paired sample $\mathrm{t}$ test. The limit for statistical significance was set at two tailed $\alpha=0.05$. Statistical software SPSS version 26 (IBM SPSS Statistics for Windows, Version 26.0. Armonk, NY: IBM Corp) was used for analysis.

\section{Results}

Within the sample, the age range was 1 to 18 median 4 years (mean 5.7, SD 4.3) at the time of diagnosis and the M:F ratio was 116:101 (1.15). The majority had standard risk disease and almost $80 \%$ remain in continuous remission (Table 1). The interval from diagnosis to December 31, 2020 was 5.7 to 21.1 median 14.7 years (mean 14.5, SD 4.2).

The recipients of alendronate $(\mathrm{n}=69,32 \%)$ were 1 to 18 median 6 years (mean 7.0 SD 5.2) of age at diagnosis and the M:F ratio was 37:32 (1.19). In the group who received alendronate $41 \%$ had high risk disease compared to $35 \%$ in the other group. Alendronate was administered for a mean (SD) of 87.1 (67.3) weeks; median 77, range 4 to 387 weeks. Two subjects were excluded from this analysis of duration of administration; one with Noonan syndrome received the drug for 6.5 years on the advice of an endocrinologist and another received prescriptions for 7.5 years but was felt to be largely non-compliant and had a LS aBMD Z score after this interval of -2.0. Both subjects were retained in the analysis of efficacy. LS aBMD prior to administration in the entire group of recipients is shown in Table 3.

SAFETY: Our experience with IV pamidronate in children with ALL has been reported. ${ }^{10}$ The side effects, including hyperpyrexia, were such that we abandoned the use of this agent. Alendronate was tolerated well overall and adherence was very high as a result of the schedule of administration. None of the participants experienced an acute phase reaction and only a few reported mild and short-lived gastrointestinal symptoms which did not interfere with compliance. However, one subject was truly intolerant, with symptomatic esophagitis, and so was withdrawn from further analyses.

Among those who received a course of alendronate, $7 / 68$ (10.3\%) relapsed, none during chemotherapy, of whom two died compared to 19/89 (21.3\%), four during post-induction chemotherapy with four deaths, in those who did not receive the drug and were not lost to follow up. The difference in relapse rates is not statistically significant $(\mathrm{p}=0.06)$. An additional child who received alendronate was diagnosed with ALL 16 years after the original diagnosis. The phenotype was demonstrably different from that of the primary disease and so the new disease was deemed to be a second cancer, recognizing that this is a rare event. ${ }^{29,30}$

\section{EFFICACY}

The interval from completion of the course of alendronate to the next DXA examination was a mean (SD) of 100.5 (86.5) weeks; median 79, range -30 to 465 weeks. One subject's last DXA was 6 months before finishing alendronate. The LS aBMD following alendronate is shown in Table 3 and represents a statistically significant increase, whether expressed as Z score (mean gain 1.31, $\mathrm{p}<0.0001$ ) or HAW corrected Z score (mean gain $0.90, \mathrm{p}<0.0001)$. While most of the subjects - 44/68 (65\%) - still had negative $\mathrm{Z}$ scores, the majority (85\%) gained BMD and 50/68 (74\%) were within the normal range of -1.0 to +1.0 . The correlation between duration of alendronate administration and the size of the gain in BMD was not statistically significant $(\mathrm{r}=0.017, \mathrm{p}=0.891$, nor was the correlation between the interval from completion of alendronate to next DXA examination and the size of the gain in BMD $(\mathrm{r}=-0.122, \mathrm{p}=0.327)$.

A subgroup of 32 subjects had further DXA examinations, as part of their long term follow up, 5-9 years after finishing alendronate. Twenty-two of them (69\%) still had negative Z scores although only half of those were osteopenic (LS aBMD Z score $<-1.00$ ). The results of the sequential DXA examinations on this subgroup are shown in Table 4 and Figure 1. The gains in BMD between time points 1 and 2 and between time points 1 and 3 were highly statistically significant $(\mathrm{p}<0.0001)$, but there was no significant difference in BMD between time points 2 and $3(\mathrm{p}=0.090)$. There was no relationship of $\mathrm{Z}$ scores or changes in $\mathrm{Z}$ scores to age or sex. Nine subjects had further scans more than 10 years from diagnosis as part of a cross-sectional study of long-term survivors; 8 had higher BMD Z scores than prior to receiving alendronate and only one was still osteopenic with a score of -1.6. 


\section{Discussion}

Bisphosphonates are manifestly effective in the treatment of osteoporosis in adults by inhibiting osteoclastmediated bone resorption. Experience in children with low bone mineral is less extensive but generally supports the same conclusion. ${ }^{19}$ This is exemplified by a report on a small number $(n=22)$ of children with a variety of chronic illnesses associated with loss of bone mineral, none with malignant disease. ${ }^{31}$ In a randomized clinical trial (RCT) the participants received either oral alendronate weekly or placebo for one year. Volumetric bone density of the lumbar spine increased significantly in the experimental group but not in those receiving placebo. Likewise, the cross-sectional moment of inertia per unit length of the femoral shaft - an estimate of mechanical strength - increased significantly only in the experimental group. ${ }^{31}$

A recent report from the Cincinatti Children's Hospital Medical Center is of a 7 year retrospective chart review describing the outcomes of intravenous bisphosphonate infusions in patients less than 21 years of age. ${ }^{32}$ Among the patients who were excluded were all of those $(\mathrm{N}=12)$ who had received cancer chemotherapy, but 29 of the study sample $(\mathrm{N}=123)$ were categorized as having glucocorticoid-induced osteoporosis. In the 42 patients who had at least two DXA examinations available for comparison there was a significant gain in LS aBMD) at one year after bisphosphonate infusion.

In the non-randomized study reported here, involving only children with ALL $(\mathrm{n}=68)$ on active therapy who received oral alendronate weekly for a shorter time than in the RCT reported by Rudge et al. ${ }^{31}$ LS aBMD increased significantly as measured by Z score with and without HAW correction. The sequential results from DXA examinations are indicative of a short-term gain in LS aBMD from the administration of alendronate, blunting an otherwise downward trajectory. These changes occurred at an age when bone mass is increasing in healthy children, adolescents and young adults. In a population of healthy Caucasian subjects in Ohio, who were studied serially by DXA between the ages of 8-30 years, ${ }^{33}$ the rate of accumulation of bone mass began to slow in mid to late teens in females $(\mathrm{N}=343)$ with peak BMD attained in the early 20s, while in males $(\mathrm{N}=312)$ the corresponding rate began to slow in late teens to early 20 s with peak BMD attained by mid to late $20 \mathrm{~s}$. Comparable Canadian data have been reported. ${ }^{34}$ Achieving a normal peak bone mass by age 30 is important in the prevention of osteoporosis in older adult life. ${ }^{35}$ Consequently, rendering the subjects in this study osteopenic from the treatment of ALL, despite a temporary and only partial reprieve by the administration of alendronate, has put them at risk of fragility fractures in later life. In a separate study from our centre, ${ }^{36}$ LS aBMD during maintenance/continuation therapy of ALL was shown to be predictive of later fractures in children who did not receive a bisphosphonate. The results in the small number of subjects $(\mathrm{n}=9)$ reported here, who were studied more than a decade after diagnosis, are intriguing, being suggestive of late improvement in LS aBMD, but longitudinal examination of a larger cohort will be required to evaluate this observation. A recent Dutch-Canadian collaborative study has demonstrated the predictive value of age and weight at diagnosis in relation to LS aBMD and to subsequent development of symptomatic fractures in children with ALL, ${ }^{37}$ complementing our established practice of HAW correction of Z scores for aBMD of the lumbar spine. ${ }^{28}$

The results of bone morphometry and measures of bone strength, determined by peripheral quantitative computed tomography (pQCT) $)^{38}$ in our study of long term survivors of ALL (more than 10 years from diagnosis), have been reported separately. ${ }^{39}$ There were no statistically significant differences in any of 19 metrics between those who had received bisphosphonate $(n=14$, pamidronate in 5$)$ and those who had not $(\mathrm{n}=58)$. This may represent adaptive restructuring of trabecular bone by thickening of a reduced number of trabeculae to maintain bone strength. ${ }^{40}$ Again, the improvement in LS aBMD following discontinuation of long-term steroid therapy has been reported in young adults with sarcoidosis. ${ }^{41}$

There have been few prior studies of bone by pQCT in children with ALL. Brennan et al. in the UK reported on 53 survivors on average almost 5 years after completion of treatment which did not include cranial radiation. ${ }^{42}$ There was no deficit in LS aBMD but reduced BMD in the trabecular bone of the distal radius was revealed. More recently, a group of 50 survivors in the US were studied within two years of completing treatment without cranial radiation, and again one year later. ${ }^{43} \mathrm{~A}$ large group of healthy subjects afforded the provision of $\mathrm{Z}$ scores. Initial deficits were shown in both trabecular and cortical BMD in the 
tibia. Subsequent changes in the cortical outcomes varied by the duration of the interval since completion of therapy. Interestingly, there were no associations of the outcomes with leukemia risk category, total glucocorticoid dose or antimetabolite therapy.

With respect to the short-term safety of oral alendronate, this has been assessed in detail in randomized clinical trials conducted in young people. In a study of 32 adolescents with anorexia nervosa who received the drug or placebo daily for one year, the authors stated "We found alendronate to be well tolerated and safe". ${ }^{44}$ A more recent trial in 139 children with osteogenesis imperfecta, involving daily oral alendronate for two years, prompted the authors' observation "Importantly, ALN was associated with few adverse events. In particular gastrointestinal symptoms were not more common in patients receiving ALN than in those receiving placebo". ${ }^{45}$ In a systematic review of bisphosphonates in children and adolescents with secondary osteoporosis Ward and colleagues noted that oral alendronate appeared to be well tolerated for intervals up to 3 years. ${ }^{46}$ A report of the use of oral alendronate in post-menopausal osteoporosis stated that the drug was efficacious and well tolerated over a 10 year period. ${ }^{47}$ In our non-randomized study gastrointestinal symptoms were infrequent and minor in degree, with one exception, and no other adverse sequelae were recorded. The retrospective study by Nasomyont and colleagues ${ }^{32}$ determined that "In 468 patient years of bisphosphonate exposure there were no reports of ONJ (osteonecrosis of the jaw) or AFF (atypical femoral fracture) in medical record." In a systematic review published in 2020 no cases of ONJ in children were identified. $^{48}$

The other safety issue addressed in our study is that of the relationship of bisphosphonates to the risk of cancer, in this instance relapse of ALL. We are not aware of this having been studied previously in children. By contrast, it has been a topic of considerable interest in the context of adults. Pre-clinical data and some, but not all, clinical trials suggest an anti-neoplastic effect of bisphosphonates. ${ }^{49}$ A recent study using murine models of ALL showed a significantly shorter survival in animals receiving zoledronic acid, a third generation bisphosphonate, as well as chemotherapy compared to those receiving chemotherapy alone, but this was not seen using xenografts of human B precursor and T cell ALL. ${ }^{50}$

A large study in the UK, using data extracted from the General Practice Research Database, examined 41,826 subjects who had been exposed to oral bisphosphonates and an equal number of matched controls, ${ }^{51}$ investigating the risk of developing cancer overall as well as specific neoplasms. It was reported that there was a significant protective effect in general and specifically in relation to breast and colorectal cancers. No such effect was evident for leukemias and lymphomas, but in no instance was the risk of cancer increased. In comparison, the results of a population-based cohort study in South Korea, using the National Health Insurance Services database, showed no association between exposure to oral alendronate or residronate and the risk of breast, ovarian or cervical cancer. ${ }^{52}$ This study involved 14,847 users and 204,525 unmatched controls.

A systematic literature review of the prognostic effect of bisphosphonate exposure in adult patients with a solid tumor found improved overall survival as well as cancer-specific and recurrence-free survival. ${ }^{53}$ For individual cancer types there was an apparent benefit in overall survival for patients with gastro-esophageal cancer and longer cancer-specific survival in those with breast cancer. In a separate study, exposure to alendronate was associated with a lower risk of bone metastases in osteoporotic women with breast cancer. ${ }^{54}$

Against this background our finding that there was no increased frequency of relapse in children receiving oral alendronate while undergoing active therapy for ALL may offer a measure of reassurance. Whether there was a protective effect is intriguing - the proportion of patients who relapsed after receiving alendronate was less than $50 \%$ of that in those who did not receive the drug, and more patients who received alendronate had high risk disease - but cannot be addressed with confidence. However, in a recent report, ${ }^{55} \mathrm{~B}$ cell ALL cells were shown to mediate resorption of trabecular bone by a RANK-RANKL (receptor activator of nuclear factor $\mathrm{kB}$ ligand) mechanism through activation of osteoclasts. The authors postulated that combining an anti-resorptive agent with chemotherapy may reduce the risk of relapse by disrupting this B cell activity.

This was a retrospective cohort study with a small number of patients. Other limitations are the variability 
in the duration of alendronate administration, although this reflects variation in the timing of responsiveness to this medication, and the lack of reliable information on fractures. A much larger longitudinal study with well-matched controls, perhaps in the form of a randomized trial to examine the efficacy of a bisphosphonate in osteopenic children with ALL, would be valuable.

Conflict of Interest statement. The authors have no conflicts of interest to declare

Acknowledgements. We acknowledge the contributions of Jo-Ann Fowler BScN and Sarah Beasley BScN to the collection of data from clinical records.

Figure legend. Sequential lumbar spine bone mineral density $\mathrm{Z}$ scores in children and adolescents with acute lymphoblastic leukemia who received alendronate.

\section{References}

1. Rabin KR, Gramatges MM, Margolin JF, Poplack DG. Acute lymphoblastic leukemia. In- Pizzo PA and Poplack DG, eds. Principles and Practice of Pediatric Oncology, $7^{\text {th }}$ Edition. Philadelphia, Wolters Kluwer 2016 pp 463-497.

2. Halton J, Gaboury I, Grant R et al. Advanced vertebral fracture among newly diagnosed children with acute lymphoblastic leukemia: Results of the Canadian Steroid Associated Osteoporosis in the Pediatric Population (STOPP) research program. J Bone Miner Res 2009; 24: 1326-1334.

3. Halton JM, Atkinson SA, Fraher L et al Mineral homeostasis and bone mass at diagnosis in children with acute lymphoblastic leukemia. J Pediatr 1995; 126: 557-564.

4. Halton JM, Atkinson SA, Fraher L et al. Altered mineral metabolism and bone mass in children during treatment for acute lymphoblastic leukemia. J Bone Miner Res 1996; 11: 1774-1783.

5. Sala A, Barr RD. Osteopenia and cancer in children and adolescents. The fragility of success. Cancer 2007; 109: 1420-1431.

6. Barr RD, Sala A. Osteonecrosis in children and adolescents with cancer. Pediatr Blood Cancer 2008; 50: $483-485$.

7. Ward LM, Tricco AC, Phoung P et al. Bisphosphonate therapy for children and adolescents with secondary osteoporosis. Cochrane Database Syst Rev. 2007: CD 005324.

8. Kotecha RS, Powers N, Lee S-J, Murray KJ, Carter T, Cole C. Use of bisphosphonates for the treatment of osteonecrosis as a complication of therapy for childhood acute lymphoblastic leukemia (ALL). Pediatr Blood Cancer 2010; 54: 934-940.

9. Drake MT, Clarke BL, Khosla S. Bisphosphonates: Mechanism of action and role in clinical practice. Mayo Clin Pract 2008; 83: 1032-1045.

10. Barr RD, Guo CY, Wiernikowski J, Webber C, Wright M, Atkinson S. Osteopenia in children with acute lymphoblastic leukemia: a pilot study of amelioration with Pamidronate. Med Pediatr Oncol 2002; 39: 44-46.

11. Lethaby C, Wiernikowski J, Sala A, Naronha M, Webber C, Barr RD. Bisphosphonate therapy for reduced bone mineral density during treatment of acute lymphoblastic leukemia in childhood and adolescence. A report of preliminary experience. J Pediatr Hematol Oncol 2007; 29: 613-616.

12. Nakhla M, Denker AE, Connor JD et al. Bioavailability and short-term tolerability of alendronate in glucocorticoid-treated children. Clin Ther 2011; 33: 1516-1523.

13. Black DM, Schwartz AV, Ensrud KE et al. Effects of continuing or stopping Alendronate after 5 years of treatment. The Fracture Intervention Trial Long-term Extension (FLEX): A randomized trial. JAMA 2006; 96: 2927-938.

14. Tuany Duarte N, de Oliveira Rech B, Godoy Martins I, Bertoldi Franco J, Lopez Ortega K. Can children be affected by bisphosphonate-related osteonecrosis of the jaw ? A systematic review. Int J Oral Maxillofac Surg 2020; 49: 183-191.

15. Brown JJ, Ramalingan L, Zacharin MR. Bisphosphonate - associated osteonecrosis of the jaw: does it occur in children? Clin Endocrinol (Oxf) 2008; 68: 863-867.

16. Sebestyen J, Srivastava T, Alon US. Bisphosphonates use in children. Clin Pediatr 2012; 51: 1011-1024.

17. Mundy GR, Yoneda T. Bisphosphonates as anti-cancer drugs. N Engl J Med 1998; 339: 398-400. 
18. Van Acker HH, Anguille S, Willemen Y, Smits EL, Van Tendeloo VF. Bisphosphonates for cancer treatment: Mechanisms of action and lessons from clinical trials. Pharmacol Ther 2016; 158: 24-40.

19. Ward LM, Konji VN, Ma J. The management of osteoporosis in children. Osteoporos Int 2016; 27: 2147-2179.

20. Pieters R, Schrappe M, De Lorenzo P et al. A treatment protocol for infants younger than 1 year with acute lymphoblastic leukemia (Interfant 99): an observational study and a multi-centre randomized trial. Lancet 2007; 370: 240-250.

21. Vrooman LM, Neuberg DS, Kutok JL et al. Post induction dexamethasone and individualized dosing of Escherichia coli L-asparaginase each improve outcome of children and adolescents with acute lymphoblastic leukemia: results from a randomized study - Dana Farber Cancer Institute ALL consortium Protocol 00-01. J Clin Oncol 2013: 31:1202-1210.

22. Place AE, Stevenson KE, Vrooman LM et al. Intravenous pegylated asparaginase versus intramuscular native Estherichia coli asparaginase in newly diagnosed childhood acute lymphoblastic leukemia (DFCI 05-001): a randomized open label phase 3 trial. Lancet Oncol 2015: 16: 1677-1690.

23. Silverman L, Blonquist TM, Hunt SK et al. Randomized study of pegasparaginase (SS-PEG) and calasparagase pegol (SC-PEG) in pediatric patients with newly diagnosed acute lymphoblastic leukemia or lymphoblastic lymphoms: results of DFCI ALL Consortium Protocol 11-001. Blood 2016; 128 (22): 175 (abstract).

24. Halton JM, Atkinson SA, Fraher L et al. Altered mineral metabolism and bone mass in children during treatment for acute lymphoblastic leukemia. J Bone Miner Res 1996; 11: 1774-1783.

25. Pierobon J, Webber CE, Nayiager T, Barr RD, Moran GR, Gulenchyn KY. Radiation doses originating from diagnostic procedures during the treatment and followup of children and adolescents with malignant lymphoma. J Radiol Prot 2011; 31: 83-93.

26. Sala A, Webber CE, Morrison J, Beaumont LF, Barr RD. Whole-body bone mineral content, lean body mass, and fat mass measured by dual-energy x-ray absorptiometry in a population of normal Canadian children and adolescents. Can Assoc Radiol J 2007; 58:46-52.

27. Webber CE, Beaumont LF, Morrison J, Sala A, Barr RD. Age-predicted values for lumbar spine, proximal femur and whole-body bone mineral density: Results from a population of normal children aged 3 to 18 years. Can Assoc Radiol J 2007; 58: 37-45.

28. Webber CE, Sala A, Barr RD. Accounting for body size deviations when reporting bone mineral density variables in children. Osteoporos Int 2009; 20: 113-121.

29. Zuna J, Cavé H, Eckert C et al. Childhood secondary ALL after ALL treatment. Leukemia 2006; 21: 1431-1435.

30. Hunger SP, Mullighan CG. Acute lymphoblastic leukemia in children. N Engl J Med 2015; 373: 15411552

31. Rudge S, Hailwood S, Horne A, Lucas J, Wu F, Cundy T. Effects of once-weekly oral alendronate on bone in children on glucocorticoid treatment. Rheumatology 2005; 44: 813-818.

32. Nasomyont N, Horning LN, Gordon CM, Wasserman H. Outcomes following intravenous bisphosphonate infusion in pediatric patients: A 7-year retrospective chart review. Bone 2019; 121: 60-67.

33. Lu J, Shin Y, Yen M-S, Sun SS. Peak bone mass and patterns of change in total bone mineral density and bone mineral content from childhood into young adulthood. J Clin Densitom 2016; 19: 180-191.

34. Baxter-Jones ADG, Faulkner RA, Forwood MR, Mirwald RL, Bailey DA. Bone mineral accrual from 8 to 30 years of age: An estimation of peak bone mass. J Bone Miner Res 2011; 26: 1729-1739.

35. Heaney RP, Abrams S, Dawson-Hughes B et al. Peak bone mass. Osteoporos Int 2000; 11: 985-1009.

36. Rayar MS, Nayiager T, Webber CE, Barr RD, Athale UH. Predictors of bony morbidity in children with acute lymphoblastic leukemia. Pediatr Blood Cancer 2012; 59: 77-82.

37. Verwaaijen EJ, de Groot-Kruseman HA, Pieters R et al. Developing and validating risk prediction models for symptomatic fractures and low bone mineral density in pediatric acute lymphoblastic leukemia. J Bone Miner Res 2021, Oct 5. DOI:10.1002/jbmr.4442.

38. Fonseca A, Gordon C, Barr RD. Peripheral quantitative computed tomography (pQCT) to assess bone health in children, adolescents, and young adults: A review of normative data. J Pediatr Hematol Oncol 
2013; 35: 581-589.

39. Barr R, Inglis D, Athale U, Farncombe T, Gordon C. Bone health in long-term survivors of acute lymphoblastic leukemia in childhood an adolescence. An assessment by peripheral quantitative computed tomography. Pediatr Blood Cancer 2021; 68: e29218.

40. Frost HM. On the trabecular "thickness" - number problem. J Bone Miner Res 1999; 14: 1816-1821.

41. Rizzato G, Montemurro L. Reversibility of exogenous corticosteroid-induced bone loss. Eur Resp J 1993; 6: 116-119.

42. Brennan BM, Mughal Z, Roberts SA et al. Bone mineral density in childhood survivors of acute lymphoblastic leukemia treated without cranial irradiation. J Clin Endocrinol Metab 2005; 90: 689694.

43. Mostoufi-Moab S, Brodsky J, Isaacoff EJ et al. Longitudinal assessment of bone density and structure in childhood survivors of acute lymphoblastic leukemia without cranial radiation. J Clin Endocrinol Metab 2012; 97: 3584-3592.

44. Golden NH, Iglesias EA, Jacobson MS et al. Alendronate for the treatment of osteopenia in anorexia nervosa: A randomized, double-blind, placebo-controlled trial. J Clin Endocrinol Metab 2005; 90: 31793185.

45. Ward LM, Rauch F, Whyte MP et al. Alendronate for the treatment of pediatric osteogenesis imperfecta: A randomized placebo-controlled study. J Clin Endocrinol Metab 2011; 96: 355-364.

46. Ward L, Tricco AC, Phuong P et al. Bisphosphonate therapy for children and adolescents with secondary osteoporosis. Cochrane Database Syst Rev 2007; 4: CD005324.

47. Bone HG, Hosking D, Devogelaer J-P, et al. Ten years' experience with alendronate for osteoporosis in postmenopausal women. N Engl J Med 2004; 350: 1189-1199.

48. Duarte NT, Rech B deO, Martins IS, Franco JB, Ortega KL. Can children be affaected by bisphosphonate-related osteonecrosis of the jaw ? A systematic review. Int J Oral Maxillofac Surg 2020; 49: 183-191.

49. Clezardin P. Bisphosphonates' antitumor activity: An unraveled side of a multifaceted drug class. Bone 2011; 48: 71-79.

50. Janke LJ, Kim J, Payton MA et al. Effects of zoledronic acid on osteonecrosis and acute lymphoblastic leukemia treatment efficacy in preclinical models. Pediatr Blood Cancer 2021; 68: e29183.

51. Cardwell CR, Abnet CC, Veal P, Hughes CM, Cantwell MM, Murray LJ. Exposure to oral bisphosphonates and risk of cancer. Int J Cancer 2012; 131: E717-E725.

52. Bae YE, Chang J, Park SM. Oral bisphosphonate use and the risk of female breast, ovarian, and cervical cancer: a nationwide population-based study. Arch Osteoporos 2019; 14: 41.

53. Wen DT, Xu Z, Xuan ML et al. Prognostic effect of bisphosphonate exposure for patients with diagnosed solid cancer: A systematic review with meta-analysis of observational studies. Front Oncol 2018: 8: 495.

54. Rouach V, Goldshtein I, Wolf I et al. Exposure to alendronate is associated with a lower risk of bone metastases in osteoporotic women with early breast cancer. J Bone Oncol 2018; 12: 91-95.

55. Rajakumar SA, Papp E, Lee KK et al. B cell acute lymphoblastic leukemia cells mediate RANKRANKL - dependent bone destruction. Sci Transl Med 2020; 12: eaba5942. 
Figure 1.

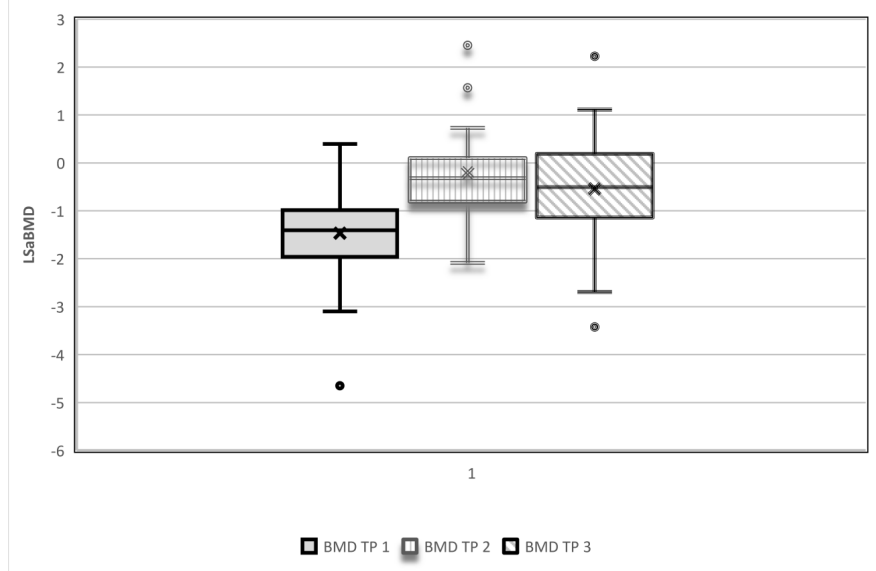

\section{Hosted file}

TABLE 1.docx available at https://authorea.com/users/406883/articles/555620-safety-andefficacy-of-alendronate-to-treat-osteopenia-in-children-during-therapy-for-acutelymphoblastic-leukemia-a-retrospective-cohort-study-of-sequential-outcomes

\section{Hosted file}

TABLE 2.docx available at https://authorea.com/users/406883/articles/555620-safety-andefficacy-of-alendronate-to-treat-osteopenia-in-children-during-therapy-for-acutelymphoblastic-leukemia-a-retrospective-cohort-study-of-sequential-outcomes

\section{Hosted file}

TABLE 3.docx available at https://authorea.com/users/406883/articles/555620-safety-andefficacy-of-alendronate-to-treat-osteopenia-in-children-during-therapy-for-acutelymphoblastic-leukemia-a-retrospective-cohort-study-of-sequential-outcomes 


\section{Hosted file}

TABLE 4.docx available at https://authorea.com/users/406883/articles/555620-safety-andefficacy-of-alendronate-to-treat-osteopenia-in-children-during-therapy-for-acutelymphoblastic-leukemia-a-retrospective-cohort-study-of-sequential-outcomes 